\title{
Review Article \\ The Impact of Extracellular Vesicle-Encapsulated Circulating MicroRNAs in Lung Cancer Research
}

\author{
Yu Fujita, ${ }^{1,2}$ Kazuyoshi Kuwano, ${ }^{2}$ Takahiro Ochiya, ${ }^{1}$ and Fumitaka Takeshita ${ }^{1}$ \\ ${ }^{1}$ Division of Molecular and Cellular Medicine, National Cancer Center Research Institute, 5-1-1 Tsukiji, Chuo-ku, \\ Tokyo 104-0045, Japan \\ ${ }^{2}$ Division of Respiratory Diseases, Department of Internal Medicine, Jikei University School of Medicine, \\ 3-19-18 Nishi-shinbashi, Minato-ku, Tokyo 105-8471, Japan \\ Correspondence should be addressed to Fumitaka Takeshita; futakesh@ncc.go.jp
}

Received 18 July 2014; Accepted 25 August 2014; Published 11 September 2014

Academic Editor: Chengfeng Yang

Copyright (C) 2014 Yu Fujita et al. This is an open access article distributed under the Creative Commons Attribution License, which permits unrestricted use, distribution, and reproduction in any medium, provided the original work is properly cited.

Lung cancer is the leading cause of cancer-related deaths. Biomarkers for lung cancer have raised great expectations in their clinical applications for early diagnosis, survival, and therapeutic responses. MicroRNAs (miRNAs), a family of short endogenous noncoding RNAs, play critical roles in cell growth, differentiation, and the development of various types of cancers. Current studies have shown that miRNAs are present in the extracellular spaces, packaged into various membrane-bound vesicles. Tumor-specific circulating miRNAs have been developed as early diagnostic biomarkers for lung cancer. Remarkably, some studies have succeeded in discovering circulating miRNAs with prognostic or predictive significance. Extracellular vesicles (EVs), such as exosomes and microvesicles, are recognized as novel tools for cell-cell communication and as biomarkers for various diseases. Their vesicle composition and miRNA content have the ability to transfer biological information to recipient cells and play an important role in cancer metastasis and prognosis. This review provides an in-depth summary of current findings on circulating miRNAs in lung cancer patients used as diagnostic biomarkers. We also discuss the role of EV miRNAs in cell-cell communication and explore the effectiveness of these contents as predictive biomarkers for cancer malignancy.

\section{Introduction}

Lung cancer remains the leading cause of cancer-related deaths in the world [1]. It is a heterogeneous disorder with two pathological types: non-small-cell lung cancer (NSCLC) and small-cell lung cancer (SCLC). Approximately $85 \%$ of all lung cancers are categorized as NSCLC. The most common pathological types of NSCLC are adenocarcinoma (30$50 \%$ ) and squamous cell carcinoma (30\%). Many therapeutic strategies, including surgery, radiotherapy, chemotherapy, and molecular target therapies, are commonly used to treat lung cancer, either alone or in combination. A majority of lung cancer patients are in advanced stages of the disease with limited treatment choices, mainly consisting of cytotoxic chemotherapeutic agents and targeted molecular therapies. Despite the development of novel targeted therapies, the prognosis for lung cancer remains poor due to drug resistance and tumor recurrence. Therefore, one of the major challenges in lung cancer research is the identification of stable biomarkers that can be routinely measured in samples that are accessible early. Biomarkers for lung cancer have raised great expectations in their clinical applications for early diagnosis, prognosis, and therapeutic responses. However, conventional serum markers, such as carcinoembryonic antigen (CEA) and squamous cell carcinoma antigen (SCC), lack sufficient sensitivity and specificity to facilitate early detection of lung cancer. Disease-driven proteomics based on mass spectrometry have serious technical limitations because of the complexity of the blood proteome. Recently, remarkable attention has been paid to cell-free nucleic acids, such as DNA, mRNA, and microRNA (miRNA), which are present at varying concentrations in the blood of cancer patients [2].

MiRNAs are endogenous, single-stranded, noncoding RNAs 19-22 nucleotides long that regulate translation through their interactions with mRNA transcripts [3]. MiRNAs are first transcribed, for the most part, by RNA polymerase II 
as a large primary miRNA (pri-miRNA) then processed by the endonuclease Drosha into a hairpin structure (precursor miRNA) and then further cleaved by the endonuclease Dicer into a single-stranded mature miRNA $[4,5]$. The mature miRNA is incorporated into a complex known as the RNA-induced silencing complex (RISC), which contains the Argonaute 2 (AGO2) and glycine-tryptophan $182 \mathrm{kDa}$ proteins (GW182). As a part of this complex, the mature miRNA regulates gene expression by binding to partially complementary sequences in the $3^{\prime}$-untranslated regions ( $3^{\prime}$ UTRs) of the target mRNAs, leading to mRNA degradation or translation inhibition [6]. MiRNAs posttranscriptionally inhibit gene expression through multiple mechanisms, all of which involve base-specific interactions with target mRNA transcripts. A single miRNA may target dozens of mRNAs and one mRNA can be regulated by multiple miRNAs. In mammals, miRNAs are assumed to regulate more than $50 \%$ of all protein-coding genes [7]. This evidence indicates that miRNAs are not only important therapeutic targets but also promising biomarkers, as their expression could reflect the information dispersed on thousands of target genes [8]. Recently, dysregulation of miRNA expression was determined to contribute to various physiological and pathological processes, such as development, differentiation, cell proliferation, apoptosis, and homeostasis [9, 10]. A number of researchers have also demonstrated that aberrant miRNA expression is related to cancer development and progression, behaving as tumor suppressors or oncogenes [11]. Some miRNAs are overexpressed or downregulated in certain cancer types. Expression patterns of miRNAs are unique to individual tissue types and differ between cancer and normal samples [12, 13]. Current data support the potential of miRNAs as biomarkers for NSCLC [13-20]. Moreover, assessment of multiple miRNA expression levels can accurately predict prognosis and survival in lung cancer [21].

Over the last few years, recent studies have shown that miRNAs are present in extracellular spaces, such as blood, urine, and saliva [2]. MiRNAs can be secreted via extracellular vesicles (EVs) and by protein-miRNA complexes, such as high-density lipoprotein (HDL) and AGO2, which is part of the RISC $[22,23]$. Circulating miRNAs are sensitive to protease treatment of plasma but are protected from plasma RNase digestion [24]. Resistance to the condition has been attributed to the above-described encapsulation and association with protein complexes $[25,26]$. Circulating miRNAs are one of the most promising next-generation biomarkers for cancer diagnosis. Moreover, the involvement of EVs in cancer biology is now of great interest. Recent studies have shown that EVs serve as versatile intercellular communication vehicles [27]. These findings have established the novel concept that EV miRNAs have potential not only as putative biomarkers but also for reflecting cancer progression. Here, we provide a perspective on the potential contribution of circulating miRNA and EV research to the development of cancer diagnosis and therapeutics.

\section{Mechanism of MicroRNA Release into Extracellular Spaces}

Intercellular miRNAs have important functions in many biological processes. Current studies in the field of lung disease have shown that miRNAs are present in extracellular spaces, such as blood, urine, and bronchoalveolar lavage fluid (BALF) [28]. Mitchell et al. firstly provided the direct evidence that circulating miRNAs derived from human prostate cancer xenografts are released into mouse circulation [23]. The release mechanism of circulating miRNAs as an active biological process remains largely unclear. The secretion of miRNAs by cells is related to the microenvironment of cells. Some possible mechanisms have been hypothesized for miRNA release: (1) active secretion of miRNAs in the form of vesicle-containing miRNAs and (2) energy-free passive leakage of cellular miRNAs from broken cells [29]. In fact, circulating miRNAs have been found to be packaged into various membrane-bound vesicles, such as exosomes and microvesicles [26] and to exist in a vesicle-free form associated with protein or high-density lipoprotein complexes [24] (Figure 1). Furthermore, miRNAs can be incorporated into apoptotic bodies [30]. Although the terms exosomes and microvesicles frequently are used interchangeably, these vesicles differ in their sources and the mechanisms of derivation and have distinct structural and biochemical properties that are likely to affect their roles [31]. Some methods for isolating circulating miRNAs from exosomes and microvesicles in human plasma have been developed, such as ultracentrifugation [32] and the ExoQuick precipitation method [33]. However, the exosomal miRNA profiles are affected by the different extracellular vesicle isolation methods [34]. In addition, it is technically difficult to fully discriminate between exosomes and microvesicles using these collection methods. Therefore, we will use the term extracellular vesicle (EV) in this review, according to the definition of the International Society for Extracellular Vesicles (ISEV), when describing studies using ultracentrifugation to isolate EVs [35]. EVs, especially exosomes, are small membrane vesicles that are released extracellularly after the fusion of multivesicular bodies (MVB) with the cell membrane [27]. On the other hand, microvesicles are vesicles shed by the plasma membrane of healthy cells [36]. The difference between these two terms is based on size of the vesicles: exosomes are in the range of $10-100 \mathrm{~nm}$ and microvesicles are in the range of $100-$ $1000 \mathrm{~nm}$ [37]. Importantly, it has been suggested that EVs carry communications between cells, allowing for cells to promote biological functions at distant sites [38]. However, they may not be the most prevalent form of circulating miRNAs. Arroyo et al. showed that the majority of serum miRNAs are present as AGO2-miRNA complexes but not within EVs [24]. Significantly, only EV-miRNAs reportedly have a function in communicating between cells and play a role in various biological processes, including immune system regulation, inflammation, and tumor development $[28,31]$. Therefore, we consider that EV-miRNAs make it possible to reflect every aspect of human physiological status and provide the advantage of being better biomarkers than other circulating miRNAs. 


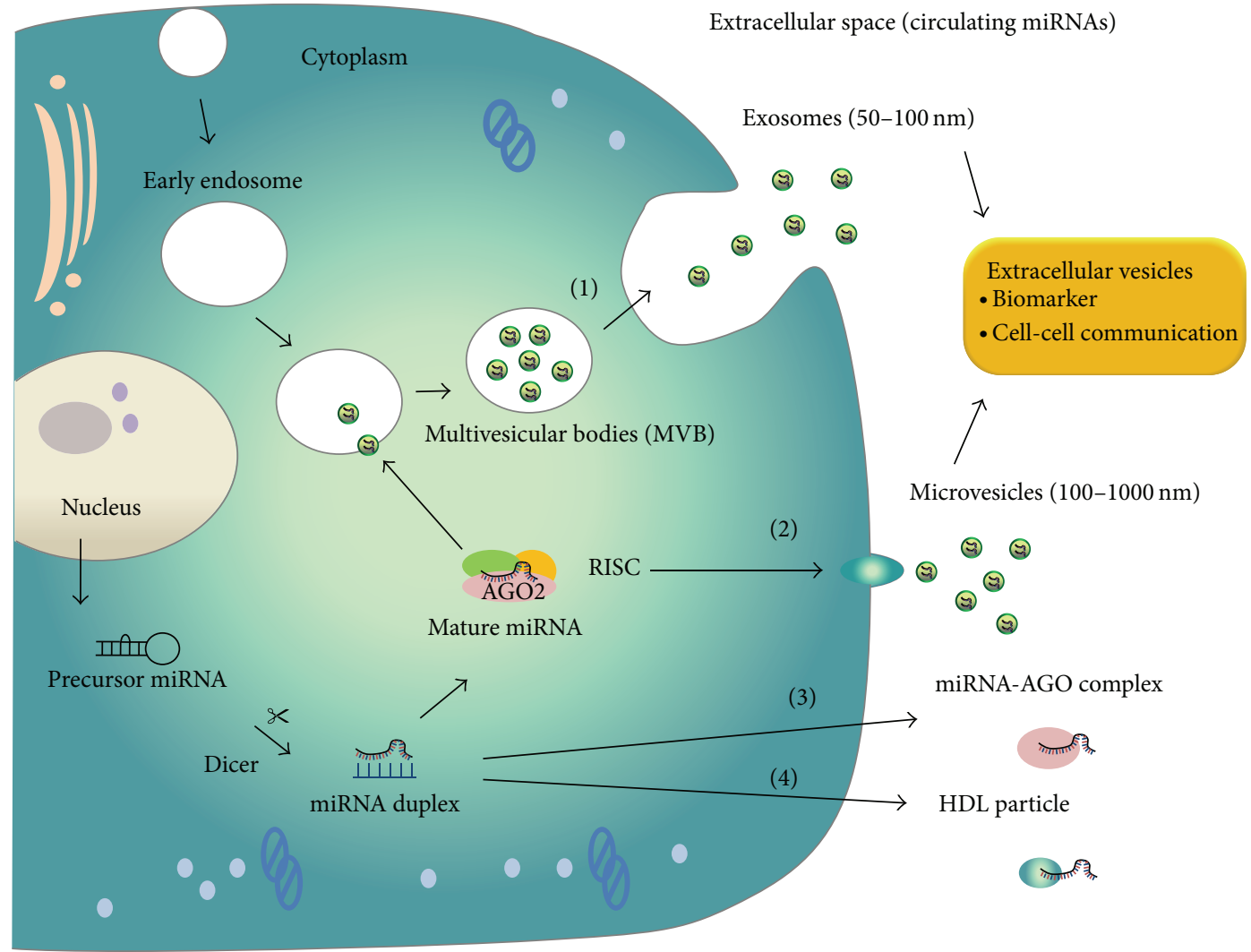

FIGURE 1: MiRNA release mechanisms into extracellular space. Precursor miRNAs are processed by ribonuclease Dicer to mature doublestranded miRNAs (miRNA duplex). One strand of the miRNA duplex is selectively loaded into the RNA-induced silencing complex (RISC), which contains the Argonaute (AGO) family protein as a core component. A fraction of miRNAs are released from living cells into the extracellular environment via the following mechanisms: (1) sorting into multivesicular bodies (MVB) and secretion via exosomes, (2) incorporating into microvesicles that are formed by the outward shedding of the plasma membrane, (3) associating with RNA-binding proteins, such as AGO2 and release of the miRNA-AGO complexes, and (4) exporting and incorporating into high-density lipoprotein (HDL) particles. Extracellular vesicle miRNAs are possibly involved in cell-cell communication.

\section{The Potential of MicroRNAs as Circulating Biomarkers in Cancer Patients}

Early cancer detection and improved therapeutic response prediction remain the major challenges in cancer research. Currently, tumor cells have been demonstrated to secrete miRNAs into body fluids [2]. With the development of detection technologies, including RT-PCR, microarray, and deep sequencing, we can screen for circulating miRNAs and generate miRNA signatures in body fluids. Circulating miRNAs are novel minimally invasive diagnostic tools for the detection and risk assessment of various types of cancer. Notably, circulating miRNAs levels in serum or plasma could correlate with cancer progression, therapeutic responses, and survival [39]. In general, miRNAs remain stable in the blood circulation. This high stability is due to their resistance to RNase activity, temperatures, extremes of $\mathrm{pH}$, and extended storage in frozen conditions [40]. This evidence suggests that miRNAs could also be used for diagnostic cancer screening or as noninvasive biomarkers for disease monitoring [41]. Blood-based tests would be more reasonable, as they have relatively low cost and can be repeated as well.
Several researches have revealed that circulating miRNA levels are higher in cancer patients than in healthy donors. Tumor-specific miRNAs (miR-21) were first reported in the serum of patients with diffuse large B-cell lymphoma (DLBCL), indicating that circulating miRNAs can be used as biomarkers to monitor the existence of cancer cells [42]. After this research, many studies have analyzed the clinical relevance of circulating miRNAs in blood for diagnosis and survival [23, 43, 44]. To date, differential expression of circulating miRNAs has been reported in cancers of the lung [44], breast [43], liver [45], kidney [46], bladder [47], and prostate [23], among others. Particularly, Mitchell et al. clearly showed that circulating miRNAs originate from tumors and are protected from endogenous RNase activity, suggesting the high potential for using circulating miRNAs as blood biomarkers for cancer [23]. Their most important advantage is the possibility for repeated measurements in a noninvasive manner. Easy access and superior stability in blood plasma and serum also add to their potential as cancer biomarkers [24, 48]. Remarkably, highly expressed circulating miRNAs from cancer patients have been reported 
TABLE 1: Circulating miRNAs as a potential biomarker for lung cancer.

\begin{tabular}{|c|c|c|c|c|}
\hline Study design & Body fluid type & MicroRNA & Detection method & Reference \\
\hline NSCLC versus normal & Serum & miR-25 and -223 & NGS, qRT-PCR & {$[44]$} \\
\hline NSCLC versus normal & Serum & miR-15b and $-27 b$ & qRT-PCR & {$[52]$} \\
\hline NSCLC versus normal & Plasma vesicles & let-7f and $\mathrm{miR}-30 \mathrm{e}-3 \mathrm{p}$ & qRT-PCR array & {$[53]$} \\
\hline Stage I/II versus IV NSCLC & Serum & miR-126 and -183 & qRT-PCR & {$[54]$} \\
\hline NSCLC versus normal & Plasma & miR-21, $-126,-210,-486-5 p$ & qRT-PCR & {$[55]$} \\
\hline NSCLC versus normal & Sputum & miR-31, -210 & qRT-PCR & {$[56]$} \\
\hline NSCLC versus normal & Sputum & miR-31, -210 & qRT-PCR & {$[57]$} \\
\hline $\begin{array}{l}\text { NSCLC versus normal } \\
\text { (metastasis stage) }\end{array}$ & Serum & miR-21 & qRT-PCR & {$[60]$} \\
\hline Prognosis of NSCLC & Serum & $\operatorname{miR}-486,-30 d,-1,-499$ & NGS, qRT-PCR & {$[41]$} \\
\hline Prognosis of NSCLC & Plasma & $\begin{array}{l}\operatorname{miR}-221,-660,-486-5 p,-28-3 p,-197 \\
-106 a,-451,-140-5 p,-16\end{array}$ & qRT-PCR array & {$[58]$} \\
\hline Prognosis of NSCLC & Serum & $\operatorname{miR}-21,-141,-200 c$ & qRT-PCR & {$[59]$} \\
\hline $\begin{array}{l}\text { Response parameter for } \\
\text { chemotherapy }\end{array}$ & Serum & $\operatorname{miR}-125 b$ & qRT-PCR & {$[61]$} \\
\hline
\end{tabular}

NGS: next generation Solexa sequencing; qRT-PCR: quantitative real-time polymerase chain reaction; miR: microRNA; NSCLC: non-small-cell lung cancer.

to return to a normal level after tumor resection. Yamamoto et al. have reported that high levels of miR-500 were found in the serum of patients with hepatocellular carcinoma, while the circulating miR-500 returned to normal levels after surgical operation in three of the patients [49]. Serum levels of upregulated miRNAs such as miR-195 and let-7a are significantly higher in preoperative plasma from breast cancer patients than after resection [50]. Yamada et al. have reported that surgical removal of urothelial carcinoma coincided with a reduction in urine miR-96 and miR-183 levels [51]. These findings have supported the idea that the level of circulating miRNAs may reflect the expression level of tumor miRNAs.

\section{Circulating MicroRNAs in Lung Cancer Patients}

Lung cancer is often diagnosed in the late stages with poor prognoses. Therefore, it is important to develop biomarkers to identify early-stage lung cancer and to predict therapeutic responses to chemotherapy or radiotherapy. Circulating miRNAs have been proposed as attractive candidates to be used as cancer biomarkers and are ideal for monitoring by screening. So far, several studies have analyzed the potential diagnostic and prognostic role of circulating miRNAs (Table 1).

Chen et al. used deep sequencing followed by qRT-PCR to identify two NSCLC-specific serum miRNAs (miR-25 and miR-223) [44]. This is the first comprehensive analysis of miRNA profiles in the serum of patients with NSCLC using Solexa sequencing technology. After this study, a lot of research aimed to identify circulating miRNAs with a diagnostic relevance in body fluids such as serum, plasma, sputum, and pleural effusion [44, 52-57]. Bianchi et al. evaluated a panel of 361 miRNAs in patients from the COSMOS trial to focus on the diagnostic accuracy of circulating miRNAs in NSCLC [52]. This study reports a combination of two differentially expressed miR-15b and miR27b capable of discriminating NSCLC from healthy donors with high sensitivity and specificity in the training set. This suggests that serum miRNAs have the promising potential to be sensitive and cost-effective biomarkers for the early detection of NSCLC. Although various miRNAs were identified as biomarkers capable of distinguishing between NSCLC patients and healthy donors, different research identified different promising miRNAs.

Recently, several groups have revealed defined serum or plasma miRNA signatures with prognostic and predictive clinical relevance [41, 58, 59]. The most comprehensive study for circulating miRNAs related to prognosis has been conducted by $\mathrm{Hu}$ et al. [41]. In a follow-up validation set of 243 NSCLC patients, the serum levels of four miRNAs (miR486, miR-30d, miR-1, and miR-499) were significantly related to a poor prognosis. When these miRNAs were combined to generate the four miRNA signatures, patients with high-risk signatures had a 9.31-fold increased hazard ratio for cancer death and a shorter median survival. Following computed tomography screening, Boeri et al. showed that 9 circulating miRNAs (miR-221, miR-660, miR-486-5p, miR28-3p, miR197, miR-106a, miR-451, miR-140-5p, and miR-16) were found to indicate a risk of lung cancer malignancy and poor prognosis [58]. These data suggest that circulating miRNAs can be released into the extracellular spaces even several years before the clinical manifestation of lung cancer. Recently, a significant correlation was reported between serum miR-21 expression and clinicopathological factors of NSCLC patients 
[60]. High serum levels of this miRNA were significantly related to lymph node metastasis, advanced clinical staging, and a poor prognosis. Moreover, vesicle-related miRNAs have also been quantified and found to be significant cancer prognosis biomarkers (let-7f and miR-30e-3p) [53].

In advanced NSCLC patients receiving cisplatin-based chemotherapy, high expression level of circulating miR-125b was a diagnostic and prognostic marker [61]. In addition, the high serum level of miR-125b was shown to be associated with poor sensitivity to neoadjuvant chemotherapy in various types of cancer $[61,62]$. This data strongly implied the feasibility of miRNAs as noninvasive or circulating biomarkers for not only the early detection of lung cancer but also its chemoresistance.

Despite promising developments in this research field, circulating miRNAs as biomarkers for cancer need to be sufficiently investigated to validate their potential. Actually, it is unclear from which types of cells the diagnostically relevant miRNAs are derived. It cannot be excluded that circulating miRNAs might be derived from a variety of cell types, such as blood cells, tumor-associated fibroblasts, endothelial cells, and various immune cells residing in the tumor microenvironment. Therefore, we must analyze the miRNA profiles expressed by noncancerous cells, which could reflect specific inflammatory or immune-modulatory processes that occur during carcinogenesis.

\section{Extracellular Vesicle-Encapsulated Circulating MicroRNAs in Cancer Progression}

EV miRNAs are derived from normal and tumor cells in body fluids as an active secretion mechanism and can mediate cell-cell communication. EVs containing mRNA, miRNAs, and angiogenic proteins released by cancer cells have been revealed [63]. The Swedish group first showed the remarkable discovery that miRNAs are contained inside EVs, which are lipoprotein complexes, including small membrane vesicles of endocytic origin [26]. They demonstrated that mouse and human mast cell-derived EVs contain RNA and miRNA and that RNA from mast cell EVs is transferable to other mouse and human mast cells. In 2010, three groups reported that EV miRNAs are transferred to recipient cells and suppress the expression of target genes [64-66]. Over the last decade, EVs have become the next focus of intensive scientific research as novel mediators of intercellular communication. Recent studies have shown the deep involvement of tumor cellderived EVs in cancer progression. Moreover, EVs secreted from the surrounding cells have also been reported to regulate tumor pathology [67]. Kosaka et al. reported that EV miRNA-210 released by metastatic breast cancer cells entered endothelial cells and suppressed the expression of its target genes, which resulted in enhanced angiogenesis [68]. Rana et al. reported that EV miR-494 and miR-542-3p derived from rat adenocarcinoma cells modulate premetastatic organ cells predominantly through transferred miRNA, where miRNA from a metastasizing tumor prepares premetastatic organ stroma cells for tumor cell hosting [69]. Fabbri et al. found that EV miR-21 and miR-29a can induce a Toll-like receptor (TLR)- mediated prometastatic inflammatory response by binding to TLRs regulating for the tumor microenvironment [70]. Recently, Zhou et al. found that EV miR-105 can destroy vascular endothelial barriers to promote brain metastasis [71]. These data suggest the deep involvement of cancer cell-derived EVs in cancer malignancy such as metastasis. Therefore, EV miRNAs have potential not only to be putative biomarkers but also to reflect cancer progression. For this reason, we suggest that $\mathrm{EV}$ miRNAs may have the potential of prognostic biomarkers to predict clinical outcome of lung cancer patients. So far, there are few reports that vesiclefree forms associated with protein or HDL complexes can mediate the expansion of cancer malignancy in an autocrine or paracrine manner [25]. The functionality of EV miRNAs in the recipient cells has opened up a new avenue of cellcell communication in cancer biology, including lung cancer research.

\section{Conclusions}

Circulating miRNAs are promising lung cancer biomarker candidates (Figure 2). Specific tumor-associated circulating miRNA signatures will have to be developed as early biomarkers for lung cancer. Remarkably, some studies have succeeded in discovering circulating miRNAs with prognostic or predictive significance. In addition, it was reported that some circulating miRNAs are strongly associated with distant metastasis, disease stage, and survival. Regarding this point, we consider that only EV miRNAs may contribute to the management of cancer progression. Therefore, EV miRNAs will be suitable predictive biomarkers for cancer malignancy metastasis and prognosis. We emphasize that it is important to investigate the role of $\mathrm{EV}$ miRNAs in cell-cell communication and to explore the effectiveness of these molecules as biomarkers in lung cancer research. As we have shown, circulating miRNAs have been found to be packaged into various membrane-bound vesicles, such as exosomes, microvesicles, and protein complexes. It is technically difficult to completely discriminate between these vesicles using simple collection methods; therefore, we need to collect vesicles using ultracentrifugation when we research the biology of EVs. Gaining deeper understanding of the methods of circulating miRNA detection and analysis will help to clarify the information carried by circulating miRNAs and lead to novel therapeutic approaches targeting the circulating miRNAs.

\section{Conflict of Interests}

The authors declare no competing financial interests. 


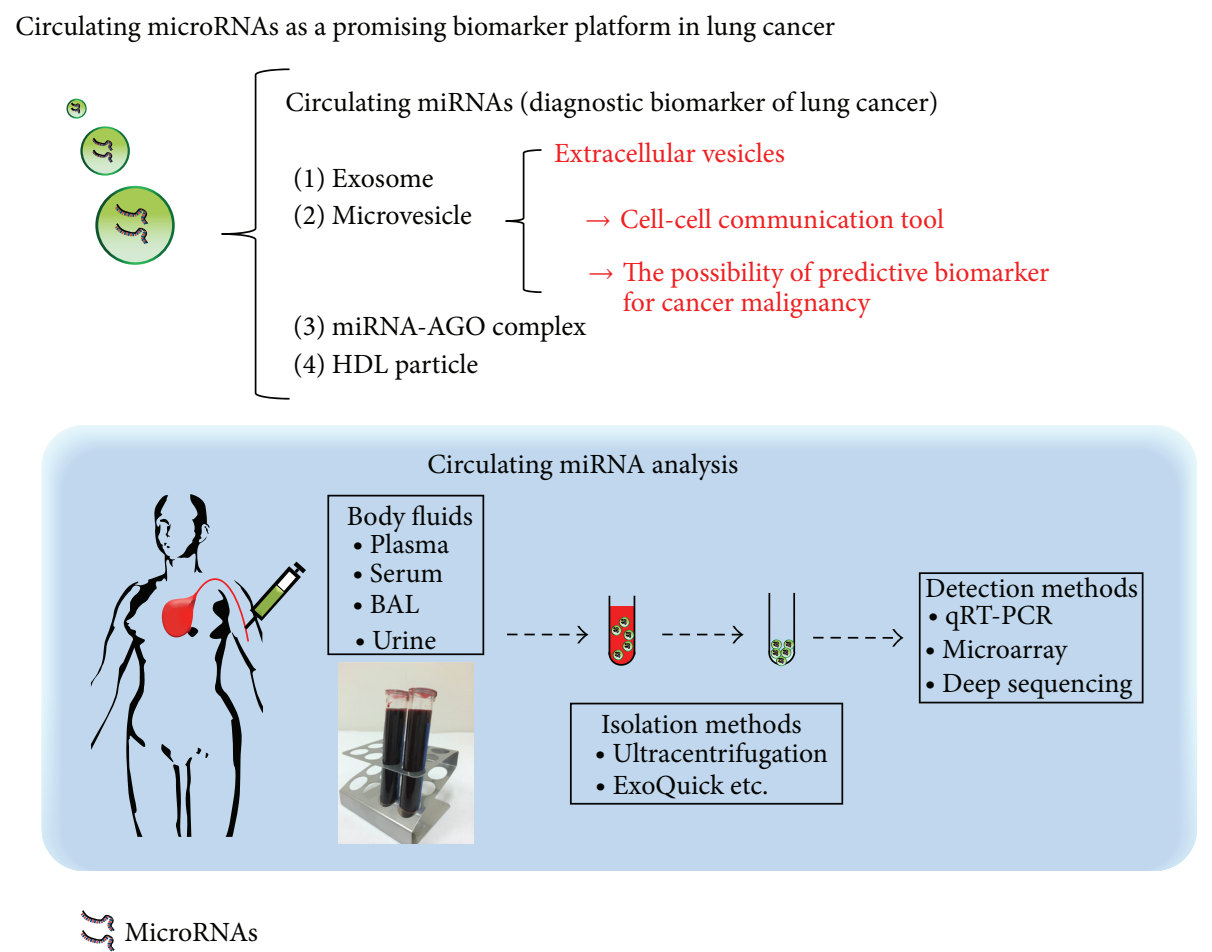

FIGURE 2: Circulating microRNAs as a promising biomarker platform in lung cancer. Circulating miRNAs have been found to be packaged into various membrane-bound vesicles, such as exosomes and microvesicles and to exist in a vesicle-free form associated with protein or high-density lipoprotein complexes. Extracellular vesicles (EVs), such as exosomes and microvesicles, are recognized as novel tools for cellcell communication and might be predictive biomarkers for cancer malignancy. EVs that circulate in body fluids can be readily recovered using several existing isolation methods and analyzed by various detection methods.

\section{Acknowledgments}

This work was supported in part by a grant-in-aid for the Third-Term Comprehensive 10-Year Strategy for Cancer Control of Japan; Project for Development of Innovative Research on Cancer Therapeutics (P-Direct); Scientific Research on Priority Areas Cancer; Scientific Research on Innovative Areas ("functional machinery for noncoding RNAs") from the Japanese Ministry of Education, Culture, Sports, Science, and Technology; the National Cancer Center Research and Development Fund (23-A-2, 23-A-7, 23-C-6,); the Program for Promotion of Fundamental Studies in Health Sciences of the National Institute of Biomedical Innovation (NiBio); the Project for Development of Innovative Research on Cancer Therapeutics; and the Japan Society for the Promotion of Science (JSPS) through the "Funding Program for World-Leading Innovative R\&D on Science and Technology (FIRST Program)" initiated by the Council for Science and Technology Policy (CSTP).

\section{References}

[1] R. Siegel, D. Naishadham, and A. Jemal, "Cancer statistics, 2013," CA: Cancer Journal for Clinicians, vol. 63, no. 1, pp. 11-30, 2013.

[2] N. Kosaka, H. Iguchi, and T. Ochiya, "Circulating microRNA in body fluid: a new potential biomarker for cancer diagnosis and prognosis," Cancer Science, vol. 101, no. 10, pp. 2087-2092, 2010.
[3] D. Baek, J. Villén, C. Shin, F. D. Camargo, S. P. Gygi, and D. P. Bartel, "The impact of microRNAs on protein output," Nature, vol. 455, no. 7209, pp. 64-71, 2008.

[4] B. R. Cullen, "Transcription and processing of human microRNA precursors," Molecular Cell, vol. 16, no. 6, pp. 861-865, 2004.

[5] Y. Lee, C. Ahn, J. Han et al., "The nuclear RNase III Drosha initiates microRNA processing," Nature, vol. 425, no. 6956, pp. 415-419, 2003.

[6] M. V. Iorio and C. M. Croce, "MicroRNA dysregulation in cancer: diagnostics, monitoring and therapeutics. A comprehensive review," EMBO Molecular Medicine, vol. 4, no. 3, pp. 143-159, 2012.

[7] J. Krol, I. Loedige, and W. Filipowicz, "The widespread regulation of microRNA biogenesis, function and decay," Nature Reviews Genetics, vol. 11, no. 9, pp. 597-610, 2010.

[8] H. Schwarzenbach, N. Nishida, G. A. Calin, and K. Pantel, "Clinical relevance of circulating cell-free microRNAs in cancer," Nature Reviews Clinical Oncology, vol. 11, no. 3, pp. 145-156, 2014.

[9] D. P. Bartel, "MicroRNAs: genomics, biogenesis, mechanism, and function," Cell, vol. 116, no. 2, pp. 281-297, 2004.

[10] M. Inui, G. Martello, and S. Piccolo, "MicroRNA control of signal transduction," Nature Reviews Molecular Cell Biology, vol. 11, no. 4, pp. 252-263, 2010.

[11] C. M. Croce, "Causes and consequences of microRNA dysregulation in cancer," Nature Reviews Genetics, vol. 10, no. 10, pp. 704-714, 2009. 
[12] J. Lu, G. Getz, E. A. Miska et al., "MicroRNA expression profiles classify human cancers," Nature, vol. 435, no. 7043, pp. 834-838, 2005.

[13] S. Volinia, G. A. Calin, C.-G. Liu et al., "A microRNA expression signature of human solid tumors defines cancer gene targets," Proceedings of the National Academy of Sciences of the United States of America, vol. 103, no. 7, pp. 2257-2261, 2006.

[14] J. Takamizawa, H. Konishi, K. Yanagisawa et al., "Reduced expression of the let-7 microRNAs in human lung cancers in association with shortened postoperative survival," Cancer Research, vol. 64, no. 11, pp. 3753-3756, 2004.

[15] R. J. Webster, K. M. Giles, K. J. Price, P. M. Zhang, J. S. Mattick, and P. J. Leedman, "Regulation of epidermal growth factor receptor signaling in human cancer cells by MicroRNA-7," The Journal of Biological Chemistry, vol. 284, no. 9, pp. 5731-5741, 2009.

[16] M. W. Nasser, J. Datta, G. Nuovo et al., "Down-regulation of micro-RNA-1 (miR-1) in lung cancer: suppression of tumorigenic property of lung cancer cells and their sensitization to doxorubicin-induced apoptosis by miR-1," Journal of Biological Chemistry, vol. 283, no. 48, pp. 33394-33405, 2008.

[17] G. J. Hurteau, J. A. Carlson, S. D. Spivack, and G. J. Brock, "Overexpression of the microRNA hsa-miR-200c leads to reduced expression of transcription factor 8 and increased expression of E-cadherin," Cancer Research, vol. 67, no. 17, pp. 7972-7976, 2007.

[18] M. Garofalo, C. Quintavalle, G. Di Leva et al., "MicroRNA signatures of TRAIL resistance in human non-small cell lung cancer," Oncogene, vol. 27, no. 27, pp. 3845-3855, 2008.

[19] Y. Hayashita, H. Osada, Y. Tatematsu et al., "A polycistronic MicroRNA cluster, miR-17-92, is overexpressed in human lung cancers and enhances cell proliferation," Cancer Research, vol. 65, no. 21, pp. 9628-9632, 2005.

[20] Y. Fujita, F. Takeshita, K. Kuwano, and T. Ochiya, "RNAi therapeutic platforms for lung diseases," Pharmaceuticals, vol. 6, no. 2, pp. 223-250, 2013.

[21] S.-L. Yu, H.-Y. Chen, G.-C. Chang et al., "MicroRNA signature predicts survival and relapse in lung cancer," Cancer Cell, vol. 13, no. 1, pp. 48-57, 2008.

[22] R. S. Redis, S. Calin, Y. Yang, M. J. You, and G. A. Calin, "Cellto-cell miRNA transfer: from body homeostasis to therapy," Pharmacology and Therapeutics, vol. 136, no. 2, pp. 169-174, 2012.

[23] P. S. Mitchell, R. K. Parkin, E. M. Kroh et al., "Circulating microRNAs as stable blood-based markers for cancer detection," Proceedings of the National Academy of Sciences of the United States of America, vol. 105, no. 30, pp. 10513-10518, 2008.

[24] J. D. Arroyo, J. R. Chevillet, E. M. Kroh et al., "Argonaute2 complexes carry a population of circulating microRNAs independent of vesicles in human plasma," Proceedings of the National Academy of Sciences of the United States of America, vol. 108, no. 12, pp. 5003-5008, 2011.

[25] K. C. Vickers, B. T. Palmisano, B. M. Shoucri, R. D. Shamburek, and A. T. Remaley, "MicroRNAs are transported in plasma and delivered to recipient cells by high-density lipoproteins," Nature Cell Biology, vol. 13, no. 4, pp. 423-433, 2011.

[26] H. Valadi, K. Ekström, A. Bossios, M. Sjöstrand, J. J. Lee, and J. O. Lötvall, "Exosome-mediated transfer of mRNAs and microRNAs is a novel mechanism of genetic exchange between cells," Nature Cell Biology, vol. 9, no. 6, pp. 654-659, 2007.

[27] C. Théry, "Exosomes: secreted vesicles and intercellular communications," F1000 Biology Reports, vol. 3, no. 1, article 15, 2011.
[28] Y. Fujita, Y. Yoshioka, S. Ito, J. Araya, K. Kuwano, and T. Ochiya, "Intercellular communication by extracellular vesicles and their microRNAs in Asthma," Clinical Therapeutics, vol. 36, no. 6, pp. 873-881, 2014.

[29] M. Redova, J. Sana, and O. Slaby, "Circulating miRNAs as new blood-based biomarkers for solid cancers," Future Oncology, vol. 9, no. 3, pp. 387-402, 2013.

[30] A. Zernecke, K. Bidzhekov, H. Noels et al., "Delivery of microRNA-126 by apoptotic bodies induces CXCL12dependent vascular protection," Science Signaling, vol. 2, no. 100, Article ID ra81, 2009.

[31] C. Théry, M. Ostrowski, and E. Segura, "Membrane vesicles as conveyors of immune responses," Nature Reviews Immunology, vol. 9, no. 8, pp. 581-593, 2009.

[32] M. P. Hunter, N. Ismail, X. Zhang et al., "Detection of microRNA expression in human peripheral blood microvesicles," PLoS ONE, vol. 3, no. 11, Article ID e3694, 2008.

[33] J. F. Quackenbush, P. B. Cassidy, L. M. Pfeffer et al., "Isolation of circulating microRNAs from microvesicles found in human plasma," Methods in Molecular Biology, vol. 1102, pp. 641-653, 2014.

[34] K. Rekker, M. Saare, A. M. Roost et al., "Comparison of serum exosome isolation methods for microRNA profiling," Clinical Biochemistry, vol. 47, no. 1-2, pp. 135-138, 2014.

[35] S. J. Gould and G. Raposo, "As we wait: coping with an imperfect nomenclature for extracellular vesicles," Journal of Extracellular Vesicles, vol. 2, Article ID 20389, 2013.

[36] G. Raposo and W. Stoorvogel, "Extracellular vesicles: exosomes, microvesicles, and friends," Journal of Cell Biology, vol. 200, no. 4, pp. 373-383, 2013.

[37] T. Katsuda, N. Kosaka, F. Takeshita, and T. Ochiya, "The therapeutic potential of mesenchymal stem cell-derived extracellular vesicles," Proteomics, vol. 13, no. 10-11, pp. 1637-1653, 2013.

[38] C. Bang and T. Thum, "Exosomes: new players in cell-cell communication," The International Journal of Biochemistry of Cell Biology, vol. 44, no. 11, pp. 2060-2064, 2012.

[39] J. Shen, S. A. Stass, and F. Jiang, "MicroRNAs as potential biomarkers in human solid tumors," Cancer Letters, vol. 329, no. 2, pp. 125-136, 2013.

[40] S. Gilad, E. Meiri, Y. Yogev et al., "Serum microRNAs are promising novel biomarkers," PLoS ONE, vol. 3, no. 9, Article ID e3148, 2008.

[41] Z. Hu, X. Chen, Y. Zhao et al., "Serum microRNA signatures identified in a genome-wide serum microRNA expression profiling predict survival of non-small-cell lung cancer," Journal of Clinical Oncology, vol. 28, no. 10, pp. 1721-1726, 2010.

[42] C. H. Lawrie, S. Gal, H. M. Dunlop et al., "Detection of elevated levels of tumour-associated microRNAs in serum of patients with diffuse large B-cell lymphoma," British Journal of Haematology, vol. 141, no. 5, pp. 672-675, 2008.

[43] W. Zhu, W. Qin, U. Atasoy, and E. R. Sauter, "Circulating microRNAs in breast cancer and healthy subjects," $B M C$ Research Notes, vol. 2, article 89, 2009.

[44] X. Chen, Y. Ba, L. Ma et al., "Characterization of microRNAs in serum: a novel class of biomarkers for diagnosis of cancer and other diseases," Cell Research, vol. 18, no. 10, pp. 997-1006, 2008.

[45] W. Li, L. Xie, X. He et al., "Diagnostic and prognostic implications of microRNAs in human hepatocellular carcinoma," International Journal of Cancer, vol. 123, no. 7, pp. 1616-1622, 2008. 
[46] M. Redova, A. Poprach, J. Nekvindova et al., "Circulating miR378 and miR-451 in serum are potential biomarkers for renal cell carcinoma," Journal of Translational Medicine, vol. 10, no. 1, article 55, 2012.

[47] M. Hanke, K. Hoefig, H. Merz et al., "A robust methodology to study urine microRNA as tumor marker: microRNA-126 and microRNA-182 are related to urinary bladder cancer," Urologic Oncology, vol. 28, no. 6, pp. 655-661, 2010.

[48] A. Turchinovich, L. Weiz, A. Langheinz, and B. Burwinkel, "Characterization of extracellular circulating microRNA," Nucleic Acids Research, vol. 39, no. 16, pp. 7223-7233, 2011.

[49] Y. Yamamoto, N. Kosaka, M. Tanaka et al., "MicroRNA-500 as a potential diagnostic marker for hepatocellular carcinoma," Biomarkers, vol. 14, no. 7, pp. 529-538, 2009.

[50] H. M. Heneghan, N. Miller, A. J. Lowery, K. J. Sweeney, J. Newell, and M. J. Kerin, "Circulating microRNAs as novel minimally invasive biomarkers for breast cancer," Annals of Surgery, vol. 251, no. 3, pp. 499-505, 2010.

[51] Y. Yamada, H. Enokida, S. Kojima et al., "MiR-96 and miR-183 detection in urine serve as potential tumor markers of urothelial carcinoma: correlation with stage and grade, and comparison with urinary cytology," Cancer Science, vol. 102, no. 3, pp. 522529, 2011.

[52] F. Bianchi, F. Nicassio, M. Marzi et al., "A serum circulating miRNA diagnostic test to identify asymptomatic high-risk individuals with early stage lung cancer," EMBO Molecular Medicine, vol. 3, no. 8, pp. 495-503, 2011.

[53] J. Silva, V. García, Á. Zaballos et al., "Vesicle-related microRNAs in plasma of nonsmall cell lung cancer patients and correlation with survival," European Respiratory Journal, vol. 37, no. 3, pp. 617-623, 2011.

[54] Q. Lin, W. Mao, Y. Shu et al., "A cluster of specified microRNAs in peripheral blood as biomarkers for metastatic non-small-cell lung cancer by stem-loop RT-PCR," Journal of Cancer Research and Clinical Oncology, vol. 138, no. 1, pp. 85-93, 2012.

[55] J. Shen, N. W. Todd, H. Zhang et al., "Plasma microRNAs as potential biomarkers for non-small-cell lung cancer," Laboratory Investigation, vol. 91, no. 4, pp. 579-587, 2011.

[56] J. Shen, J. Liao, M. A. Guarnera et al., "Analysis of microRNAs in sputum to improve computed tomography for lung cancer diagnosis," Journal of Thoracic Oncology, vol. 9, no. 1, pp. 33-40, 2014.

[57] N. Li, J. Ma, M. A. Guarnera, H. Fang, L. Cai, and F. Jiang, "Digital PCR quantification of miRNAs in sputum for diagnosis of lung cancer," Journal of Cancer Research and Clinical Oncology, vol. 140, no. 1, pp. 145-150, 2014.

[58] M. Boeri, C. Verri, D. Conte et al., "MicroRNA signatures in tissues and plasma predict development and prognosis of computed tomography detected lung cancer," Proceedings of the National Academy of Sciences of the United States of America, vol. 108, no. 9, pp. 3713-3718, 2011.

[59] X.-G. Liu, W.-Y. Zhu, Y.-Y. Huang et al., "High expression of serum miR-21 and tumor miR-200c associated with poor prognosis in patients with lung cancer," Medical Oncology, vol. 29, no. 2, pp. 618-626, 2012.

[60] Z.-X. Wang, H.-B. Bian, J.-R. Wang, Z.-X. Cheng, K.-M. Wang, and W. De, "Prognostic significance of serum miRNA21 expression in human non-small cell lung cancer," Journal of Surgical Oncology, vol. 104, no. 7, pp. 847-851, 2011.

[61] E.-H. Cui, H.-J. Li, F. Hua et al., "Serum microRNA 125b as a diagnostic or prognostic biomarker for advanced NSCLC patients receiving cisplatin-based chemotherapy," Acta Pharmacologica Sinica, vol. 34, no. 2, pp. 309-313, 2013.

[62] H. Wang, G. Tan, L. Dong et al., "Circulating mir-125b as a marker predicting chemoresistance in breast cancer," PLoS One, vol. 7, no. 4, Article ID e34210, 2012.

[63] J. Skog, T. Würdinger, S. van Rijn et al., "Glioblastoma microvesicles transport RNA and proteins that promote tumour growth and provide diagnostic biomarkers," Nature Cell Biology, vol. 10, no. 12, pp. 1470-1476, 2008.

[64] D. M. Pegtel, K. Cosmopoulos, D. A. Thorley-Lawson et al., "Functional delivery of viral miRNAs via exosomes," Proceedings of the National Academy of Sciences of the United States of America, vol. 107, no. 14, pp. 6328-6333, 2010.

[65] Y. Zhang, D. Liu, X. Chen et al., "Secreted monocytic miR-150 enhances targeted endothelial cell migration," Molecular Cell, vol. 39, no. 1, pp. 133-144, 2010.

[66] N. Kosaka, H. Iguchi, Y. Yoshioka, F. Takeshita, Y. Matsuki, and T. Ochiya, "Secretory mechanisms and intercellular transfer of microRNAs in living cells," The Journal of Biological Chemistry, vol. 285, no. 23, pp. 17442-17452, 2010.

[67] T. Katsuda, N. Kosaka, and T. Ochiya, "The roles of extracellular vesicles in cancer biology: toward the development of novel cancer biomarkers," Proteomics, vol. 14, no. 4-5, pp. 412-425, 2014.

[68] N. Kosaka, H. Iguchi, K. Hagiwara, Y. Yoshioka, F. Takeshita, and T. Ochiya, "Neutral sphingomyelinase 2 (nSMase2)dependent exosomal transfer of angiogenic micrornas regulate cancer cell metastasis," The Journal of Biological Chemistry, vol. 288, no. 15, pp. 10849-10859, 2013.

[69] S. Rana, K. Malinowska, and M. Zöller, "Exosomal tumor microRNA modulates premetastatic organ cells," Neoplasia, vol. 15, no. 3, pp. 281-295, 2013.

[70] M. Fabbri, A. Paone, F. Calore et al., "MicroRNAs bind to Tolllike receptors to induce prometastatic inflammatory response," Proceedings of the National Academy of Sciences of the United States of America, vol. 109, no. 31, pp. E2110-E2116, 2012.

[71] W. Zhou, M. Y. Fong, Y. Min et al., "Cancer-Secreted miR-105 destroys vascular endothelial barriers to promote metastasis," Cancer Cell, vol. 25, no. 4, pp. 501-515, 2014. 

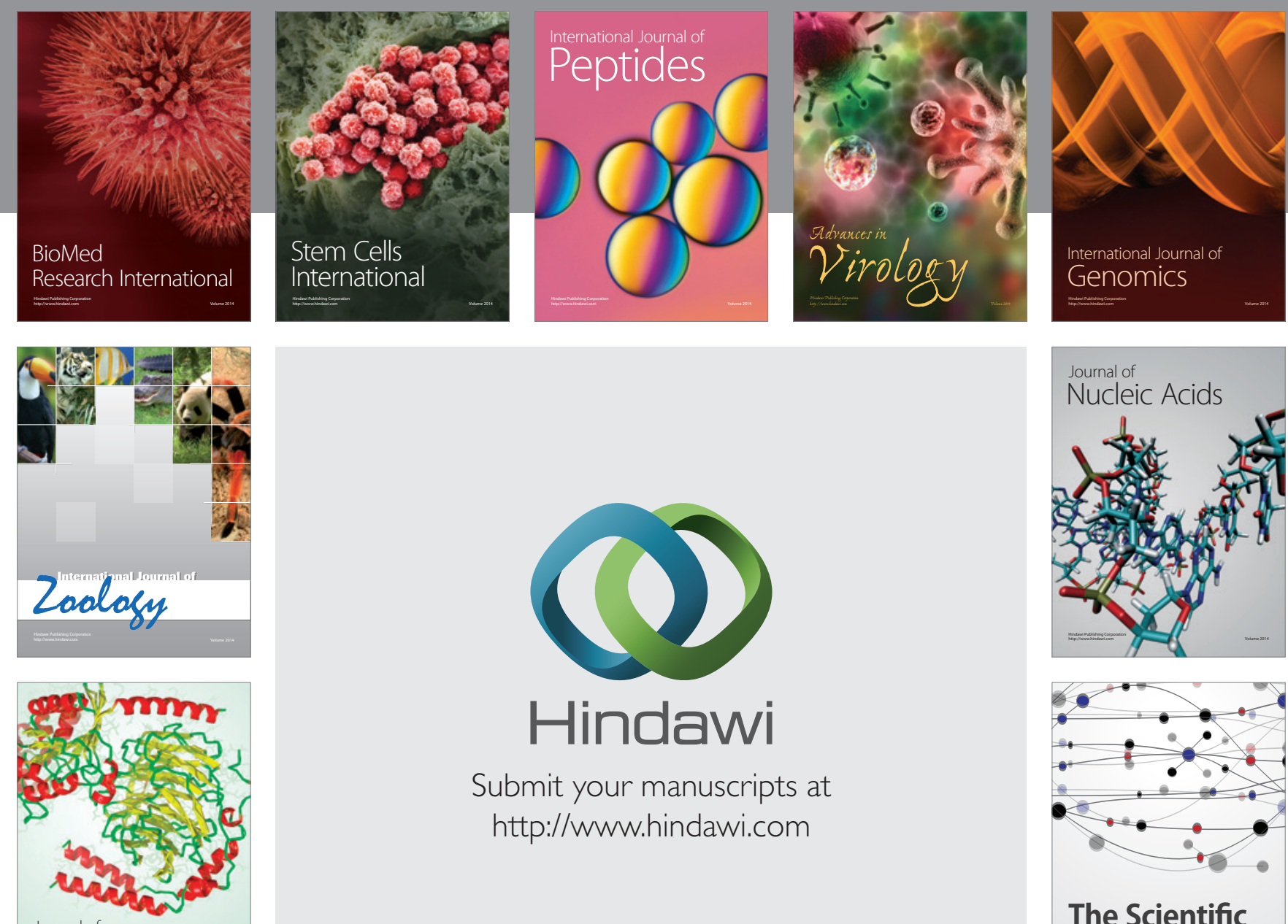

Submit your manuscripts at

http://www.hindawi.com

Journal of
Signal Transduction
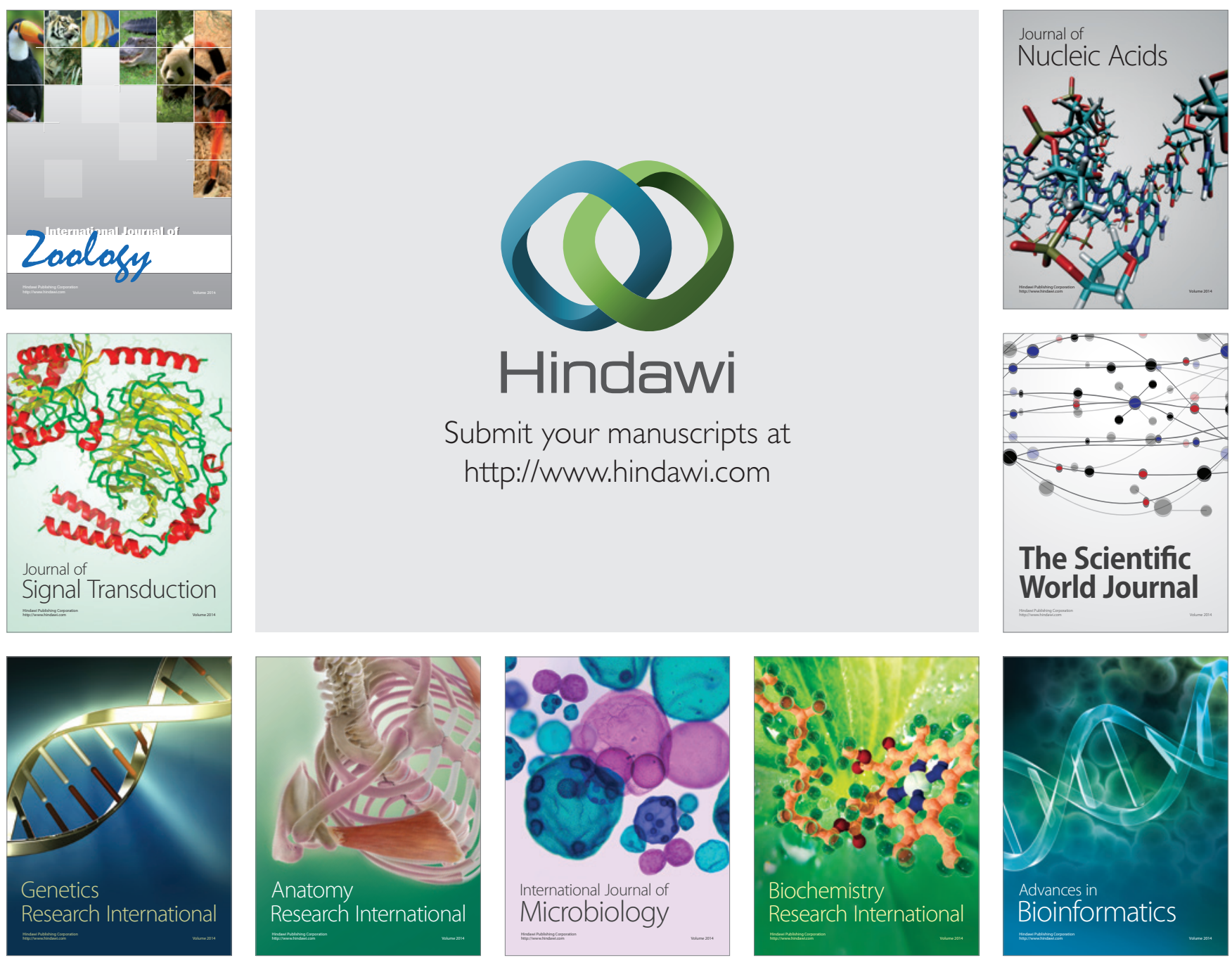

The Scientific World Journal
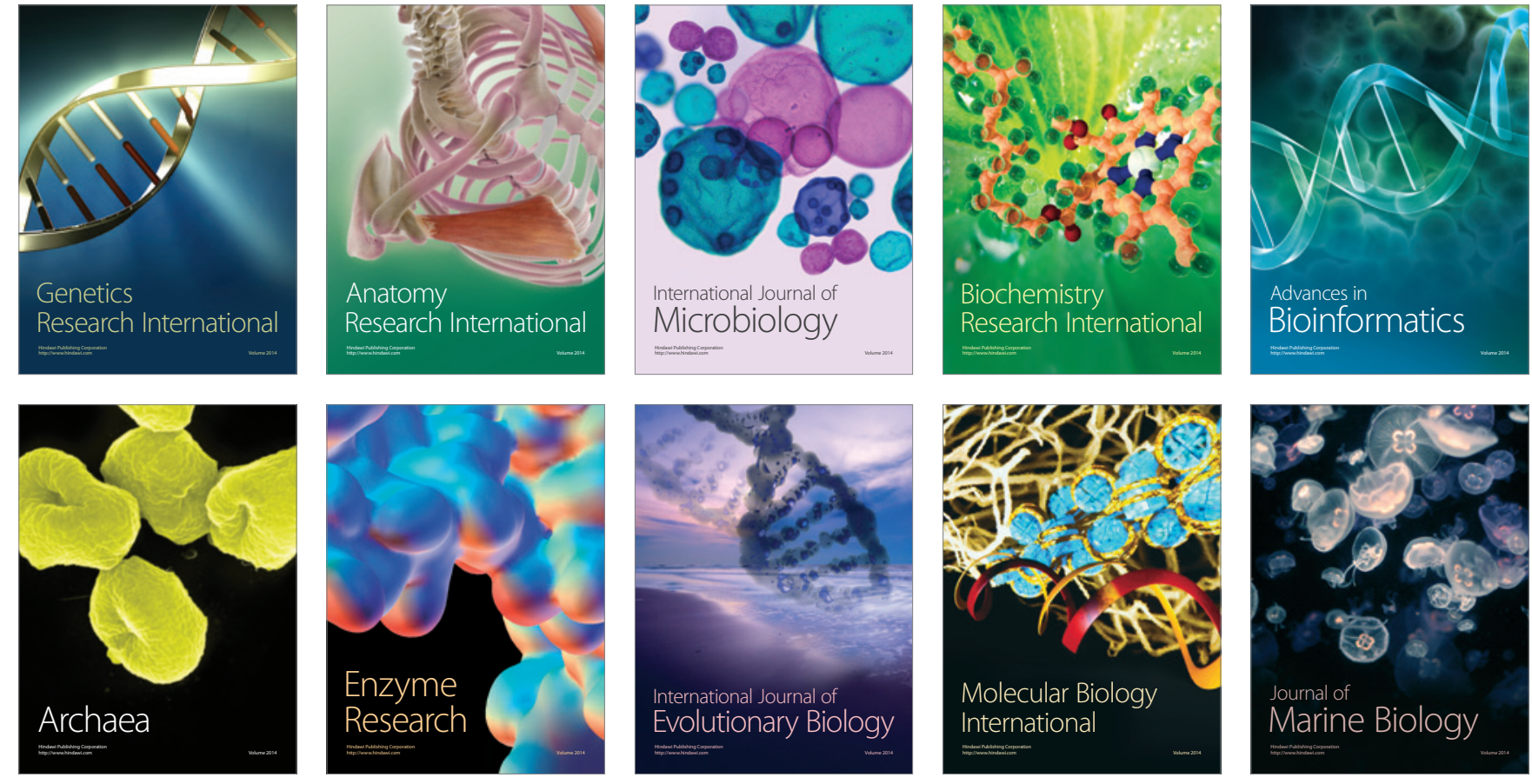